\title{
Optimal Safety of Conduction through the Purkinje-Ventricular Junction
}

\author{
P Stewart, OV Aslanidi, MR Boyett, H Zhang
}

The University of Manchester, Manchester, UK

\begin{abstract}
Slow conduction through the Purkinje-ventricular junction $(P V J)$ is thought to result in conduction block and ultimately re-entry under certain pathological conditions. The relationships between tissue structure, conduction velocity and the safety of conduction at such junctions however, are unknown. We developed an electrophysiologically detailed $3 D$ model of the PVJ and varied the Purkinje fibre thickness and intercellular coupling to determine these relationships. We found that both very fast and very slow AP conduction is unsafe, and there exists an optimal velocity providing the maximum safety of conduction through the PVJ. The conduction time delay across the junction is a natural consequence of the electrophysiological and morphological differences between the two tissue types. The delay allows the PVJ to accumulate and pass through sufficient charge to excite the adjacent ventricular tissue.
\end{abstract}

\section{Introduction}

Slow and discontinuous action potential (AP) conduction through non-uniform junctions in cardiac tissue is generally considered unsafe and pro-arrythmogenic $[1,2]$. APs conducted through the Purkinje fibre (PF) network into the ventricles determine the normal electrical activation and contraction sequence in the heart. However, conduction discontinuities due to electrophysiological and morphological differences between the thin PFs and the larger ventricles can lead to arrhythmogenic behaviour [3].

Experimentally observed time delays during discontinuous conduction from the PFs to the ventricles are considered indicative of a possible conduction block [4-6], however other studies suggest slow conduction may be indicative of higher safety [7]. The aim of this study was to use a detailed 3D model of the Purkinje-ventricular junction (PVJ) in order to determine relationships between the tissue structure, AP conduction velocity and the safety of conduction. A possibility of clinical estimates of safe propagation using ECG recordings is outlined.

\section{Methods}

The dynamics of the membrane potential in cardiac tissue can be described by the equation $[8,9]$ :

$$
\frac{\partial V}{\partial t}=\nabla \cdot D \nabla V-\frac{I_{\text {ion }}}{C_{m}}
$$

where $V$ is the membrane potential, $t$ is time, $\nabla$ is the spatial gradient operator, $D$ is the diffusion coefficient characterising the electrotonic cell-to-cell coupling by gap junctions, $C_{m}$ is the cell membrane capacitance and $I_{i o n}$ is the total membrane ionic current. Biophysically detailed models of the voltage and time dependent current $I_{i o n}$ have been developed to describe APs of the canine PF [10] and all 3 ventricular cell types (endocardial, mid-myocardial and epicardial) $[9,11]$.

The 3D wedge model has been developed earlier to incorporate details of the transmural AP heterogeneity, tissue geometry and fibre orientation of the canine left ventricular free wall [9]. In our model, we introduced a single PF strand which entered the ventricular wedge from the endocardium. Intercellular diffusion coefficients in the PF and ventricular tissues were set to reproduce the experimentally observed AP conduction velocities of $\sim 1.8$ and $0.5 \mathrm{~m} / \mathrm{s}$ respectively [12]. The free end of the PF strand was stimulated with a current pulse, resulting in AP propagation through the PVJ into the ventricular tissue. Equation (1) was solved numerically using the explicit Euler method, with space and time steps of $\Delta x=0.35 \mathrm{~mm}$ and $\Delta t=0.01 \mathrm{~ms}$, respectively. Simulations at a higher spatial resolution were also performed on an isotropic $2 \mathrm{D}$ slice with geometry similar to the central slice of the 3D model.

Safety factor $(S F)$ has been introduced as an index to quantify the robustness of conduction in cardiac tissues [13-15]. It is defined as the ratio of charge generated to charge consumed by a cell during its excitation:

$$
S F=\frac{\int_{A} I_{c} d t+\int_{A} I_{\text {out }} d t}{\int_{A} I_{\text {in }} d t} ; A \mid t \in\left[t_{1 \%}, t_{V_{\max }}\right]
$$

where, $I_{c}$ is the capacitive current of the cell, $I_{\text {out }}$ is the intercellular current that flows from the cell to its neighbours 


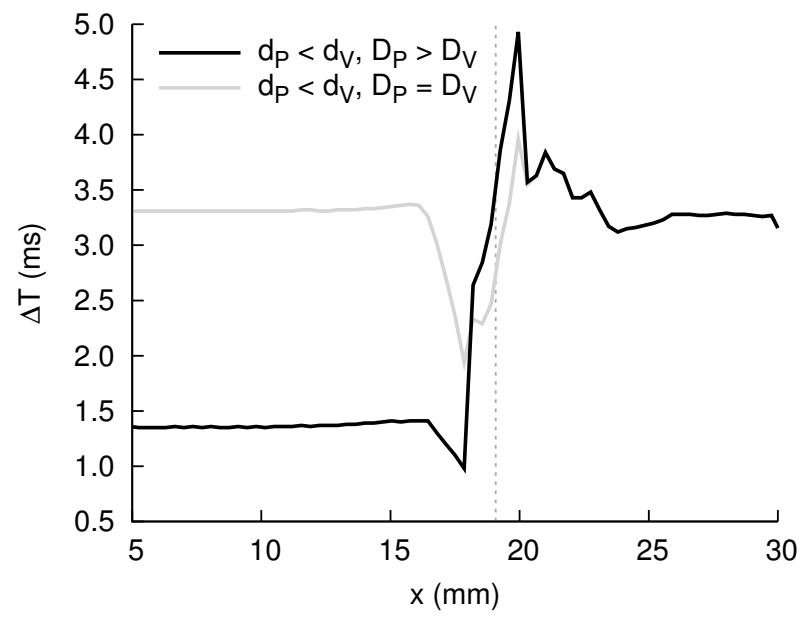

Figure 1. Conduction time delay, $\Delta T$, measured in the transmural direction, $x$. A large $\Delta T$ was produced by discontinuous AP conduction through the PVJ. Two cases illustrated: $D_{P}>D_{V}$ for an electrically and anatomically non-uniform junction, and $D_{P}=D_{V}$ showing the contribution of anatomical non-uniformities alone. Dashed line denotes location of the PVJ.

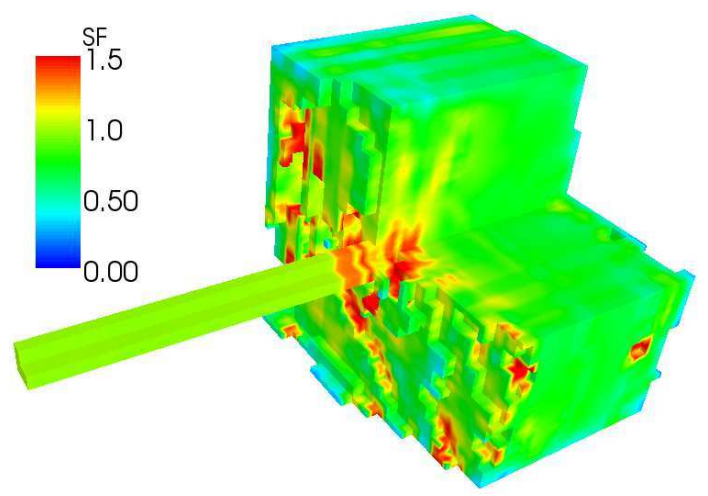

Figure 2. $S F$ distribution resultant from successful AP propagation from the $\mathrm{PF}$ into the ventricular tissue, colour coded according to palette (left).

and $I_{i n}$ is the intercellular current that flows into the cell. The domain of integration, $A$, can be defined as the period of time from the moment when $d V / d t$ at the AP wavefront reaches $1 \%$ of its maximum to the moment when $V$ reaches its maximum, $V_{\max }$ [15]. $S F>1$ indicates successful conduction, with the fraction above 1 indicating the margin of safety [14].

Thickness of the PF strand, $d_{P}$, and the diffusion coefficient of the PF, $D_{P}$, were varied in order to determine the optimal conditions (velocity and safety) of AP conduction through the PVJ. Thickness, $d_{V}$, and the diffusion coefficients ( $D_{\|}$and $D_{\perp}$ along and across fibres) of the ventricular tissue remained constant.
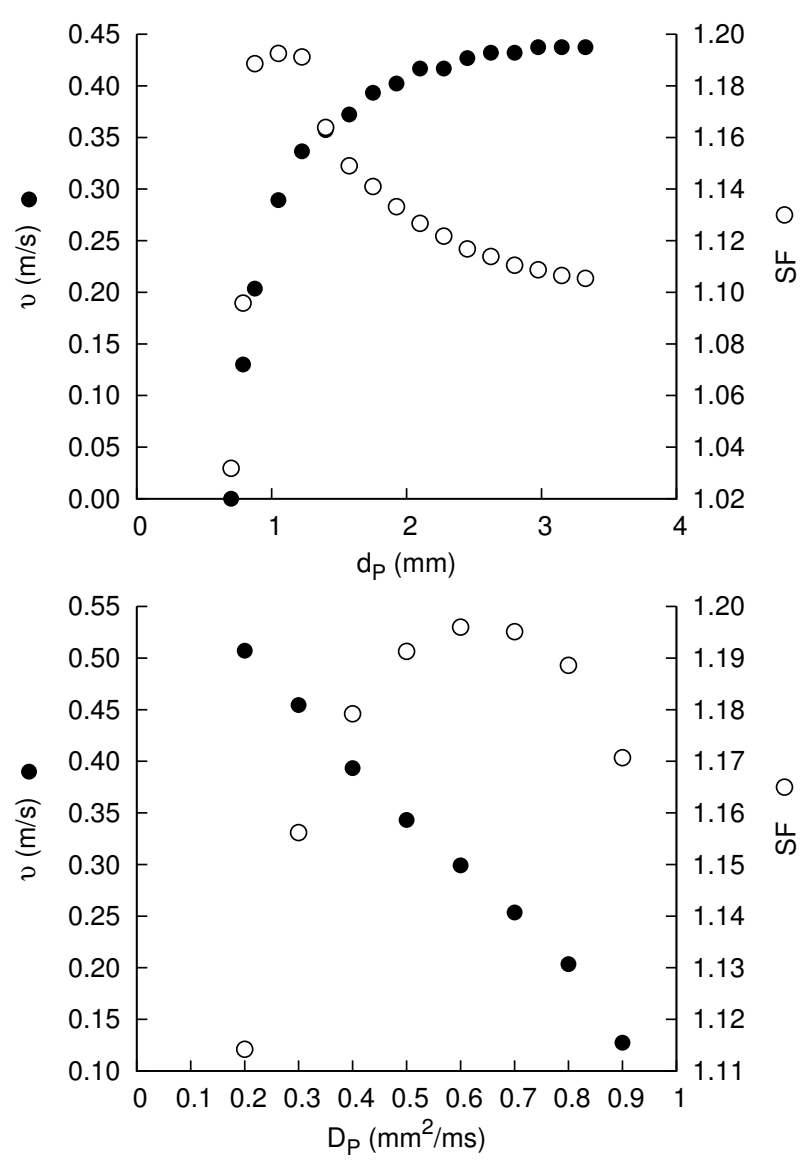

Figure 3. Relationships between non-uniformities of the PVJ, the AP conduction velocity, $v$ and safety factor, $S F$ in isotropic 2D slices. Top: dependences of $v$ and $S F$ on thickness of the PF, $d_{P}$. Bottom: dependences of $v$ and $S F$ on the diffusion coefficient of the PF, $D_{P}$. Both $v$ and $S F$ were measured at the PVJ, where $v$ reached a local minimum.

\section{Results}

Parameters $d_{P}<d_{V}$ and $D_{P}>D_{V}$ were adjusted to experimentally measured values, and reproduced experimentally observed AP conduction velocities in the PF and ventricular tissue [12]. Under such physiological conditions, AP conduction from the PF through the anatomically and electrically non-uniform PVJ was discontinuous with an abrupt increase in the time delay measured over $2 \mathrm{~mm}$, of $\sim 5 \mathrm{~ms}$ (Figure 1), consistent with experimental recordings [16]. Such a conduction time delay at the PVJ was found when non-uniformities occur in either $D, d$ or both. The conduction time delay through the PVJ resulted in a local increase in the safety factor around the junction (Figure 2), which can be due to an accumulation of charge at the PVJ before the excitation of the ventricular tissue.

As the time delay of the PVJ increased/decreased, the 


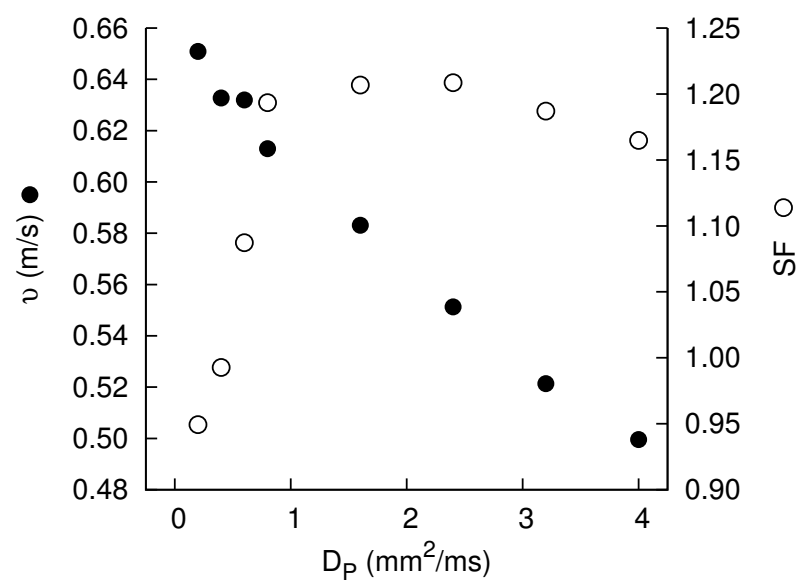

Figure 4. Relationships between the diffusion coefficient, $D_{P}$, AP conduction velocity, $v$, and safety factor, $S F$, in the anisotropic 3D model. Both $v$ and $S F$ were measured at the PVJ, where $v$ reached a local minimum.

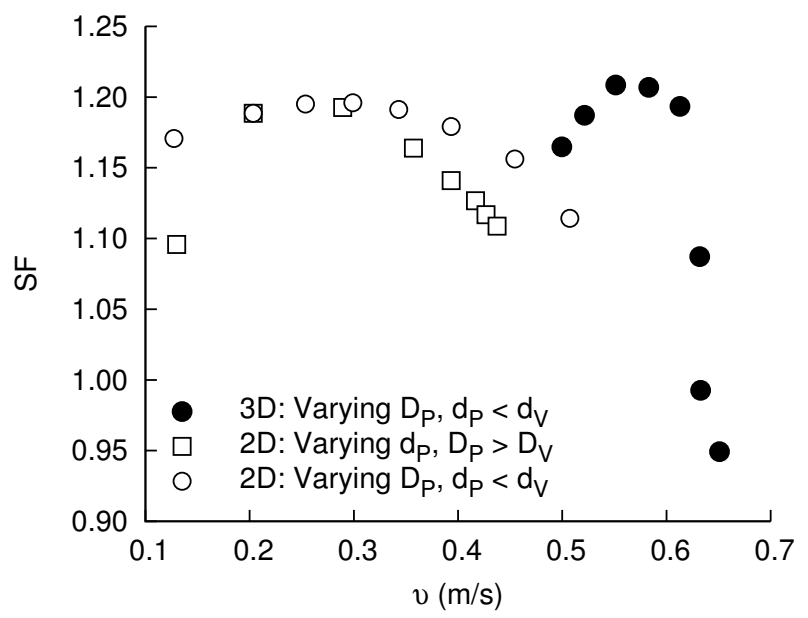

Figure 5. Relationships between the AP conduction velocity, $v$, and safety factor, $S F$, at the PVJ. Three cases illustrated: in the 3D model, $v$ is varied by changing $D_{P}$, and in the $2 \mathrm{D}$ slice, $v$ is varied by changing $d_{P}$ or $D_{P}$. In all cases, the PVJ has both electrical and anatomical nonuniformities $\left(d_{P}<d_{V}, D_{P}>D_{V}\right)$.

AP conduction velocity, $v$, decreased/increased respectively. Dependence of $v$ on varied parameters $d_{P}$ and $D_{P}$ was always monotonic (Figure 3, 4). However, dependences of $S F$ on $d_{P}$ and $D_{P}$ in 2D slices showed a maximum at $\sim 1 \mathrm{~mm}$ and $0.6 \mathrm{~mm}^{2} / \mathrm{ms}$, respectively (Figure 3 ). Resultant dependences of $S F$ on $v$ both had a maximum at $\sim 0.3 \mathrm{~m} / \mathrm{s}$ (Figure 5), which corresponds to an optimal velocity for the maximum safety of AP conduction through the PVJ. Similarly, in the 3D model, the dependence of $S F$ on $D_{P}$ had a maximum at $\sim 1.5 \mathrm{~mm}^{2} / \mathrm{ms}$ (Figure 4 ), which is greater than that in $2 \mathrm{D}$ slices due to the increased electrical load imposed by the ventricular tissue. The corresponding dependence of $S F$ on $v$ had a maximum at $\sim 0.6 \mathrm{~m} / \mathrm{s}$ (Figure 5).

\section{Discussion and conclusions}

The AP conduction time delay across the PVJ occurs as a natural consequence of the electrophysiological and morphological differences between the PF and ventricular tissue. Primarily, the much larger ventricle imposes a large electrical load on the PF, which in combination with the disparity in intercellular electrotonic coupling, leads to the long conduction time delay during AP propagation through the junction.

At the ionic level, long AP conduction time delays allowed a larger amount of charge provided by the membrane ionic currents to be accumulated by the cell capacity before passing it through the PVJ for successful excitation of the ventricular tissue. At the same time, the time delay enhanced the electrical source-to-load mismatch at the PVJ. However, at optimal velocities (Figure 5), this mismatch is not enhanced over time, such that safe conduction can occur.

We found an optimal PF thickness of $\sim 1 \mathrm{~mm}$, which corresponds to an optimal velocity of $\sim 0.3 \mathrm{~m} / \mathrm{s}$. PF bundles extracted from canine hearts have been found to measure $0.5-2.0 \mathrm{~mm}$ in diameter [17-19]. We also found optimal diffusion coefficients of $0.6 \mathrm{~mm}^{2} / \mathrm{ms}$ and $1.5 \mathrm{~mm}^{2} / \mathrm{ms}$ in $2 \mathrm{D}$ and $3 \mathrm{D}$ respectively, which corresponds to $\mathrm{PF}$ conduction velocities of around 1.4-1.8 m/s. Experimentally, $\mathrm{PF}$ conduction velocity has found to be $\sim 1.8 \mathrm{~m} / \mathrm{s}$ [12].

Our results demonstrate that both very fast or very slow conduction through the PVJ can be unsafe, and there exists an optimal velocity (and hence, PF thickness and diffusion coefficient) which provides the maximum level of safety within the physiological ranges of these parameters.

Although clinically the AP conduction velocity at the PVJ cannot be measured directly, the PR interval of the ECG may provide a means of estimating the approximate conduction safety through the PVJ. The PR interval is the length of time between atrial and ventricular depolarisation and can be attributed to conduction times through 3 main components: the atrioventricular node (AVN), the bundle of His and the PFs. Assuming there is no change in the conduction time through the AVN and bundle of His, the change in the PR interval from control (i.e. under physiological conditions), $\triangle P R$, can be used to determine relationships between $\triangle P R, v$ and $S F$ (Figure 6). A maximum in safety factor occurs within the range $\triangle P R<$ $10 \mathrm{~ms}$, which corresponds to velocities of $0.5-0.6 \mathrm{~m} / \mathrm{s}$. Beyond this range, $\mathrm{SF}$ started to decrease, and above $\triangle P R \approx$ $30 \mathrm{~ms}$ conduction becomes unsafe, indicating a possibility of conduction block and arrhythmogenic behaviour. 


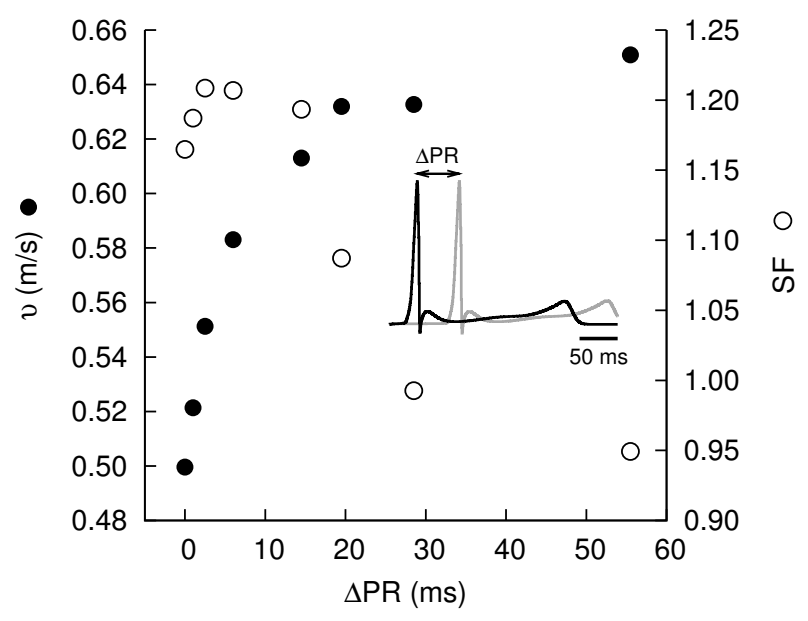

Figure 6. Relationships between changes of the PR interval in the pseudo-ECG (inset), $\triangle P R$, the AP conduction velocity, $v$, and safety factor, $S F$, in the anisotropic $3 \mathrm{D}$ model. $\triangle P R$ is measured with respect to $\mathrm{PR}$ at physiological conditions (inset, black line).

\section{Acknowledgements}

This research was supported by EPSRC (UK) DTA Studentship (PS) and BBSRC (UK) Grant No. BBS/B/1678X (OVA, MRB, HZ).

\section{References}

[1] Wit AL, Hoffman BF, Cranefield PF. Slow conduction, reentry, and the mechanism of ventricular arrhythmias in myocardial infarction. Bull N Y Acad Med 1971;47:12331234.

[2] Kucera JP, Rudy Y. Mechanistic insights into very slow conduction in branching cardiac tissue: a model study. Circ Res 2001;89:799-806.

[3] Gilmour RF, Watanabe M. Dynamics of circus movement re-entry across canine purkinje fibre-muscle junctions. J Physiol 1994;476:473-485.

[4] Overholt ED, Joyner RW, Veenstra RD, Rawling DA, Wiedmann R. Unidirectional block between purkinje and ventricular layers of papillary muscles. Am J Physiol 1984; 247:H584-595.

[5] Veenstra RD, Joyner RW, Rawling DA. Purkinje and ventricular activation sequences of canine papillary muscle. effects of quinidine and calcium on the purkinje-ventricular conduction delay. Circ Res 1984;54:500-515.

[6] Tranum-Jensen J, Wilde AA, Vermeulen JT, Janse MJ. Morphology of electrophysiologically identified junctions between purkinje fibers and ventricular muscle in rabbit and pig hearts. Circ Res 1991;69:429-437.

[7] Spach MS, Miller WT, Geselowitz DB, Barr RC, Kootsey JM, Johnson EA. The discontinuous nature of propagation in normal canine cardiac muscle. evidence for recurrent dis- continuities of intracellular resistance that affect the membrane currents. Circ Res 1981;48:39-54.

[8] Fenton FH, Karma A. Vortex dynamics in three dimensional continuous myocardium with fibre rotation: Filament instability and fibrillation. Chaos 1998;8:20-47.

[9] Benson AP, Aslanidi OV, Zhang H, Holden AV. The canine virtual ventricular wall: A platform for dissecting pharmacological effects on propagation and arrhythmogenesis. Prog Biophys Mol Biol 2008;96:187-208.

[10] Stewart P, Aslanidi OV, Zhang H. A novel mathematical model of the electrical action potential in a canine purkinje fiber cell. In Computers in Cardiology 2007, volume 34. Los Alamitos: IEEE, 2007; 363-366.

[11] Hund TJ, Rudy Y. Rate dependence and regulation of action potential and calcium transient in a canine cardiac ventricular cell model. Circulation 2004;110:3168-3174.

[12] Joyner RW, Overholt ED. Effects of octanol on canine subendocardial purkinje-to-ventricular transmission. Am J Physiol 1985;249:H1228-1231.

[13] Kucera JP, Kleber AG, Rohr S. Slow conduction in cardiac tissue, ii: Effects of branching tissue geometry. Circ Res 1998;83:795-805.

[14] Shaw RM, Rudy Y. Ionic mechanisms of propagation in cardiac tissue: Roles of the sodium and 1-type calcium currents during reduced excitability and decreased gap junction coupling. Circ Res 1997;81:727-741.

[15] Romero L, Trenor B, Ferrero JM, Saiz J. A sensitivity study of the safety factor for conduction in the myocardium. In Computers in Cardiology 2005, volume 32. Los Alamitos: IEEE, 2005; 873-876.

[16] Kanter HL, Laing JG, Beau SL, Beyer EC, Saffitz JE. Distinct patterns of connexin expression in canine purkinje fibers and ventricular muscle. Circ Res 1993;72:11241131.

[17] Reiser J, Anderson GJ. Preferential sensitivity of the left canine purkinje system to cardiac glycosides. Circ Res 1981; 49:1043-1054.

[18] Christini DJ, Riccio ML, Culianu CA, Fox JJ, Karma A, Gilmour RF. Control of electrical alternans in canine cardiac purkinje fibers. Phys Rev Letts 2006;96:104101104104.

[19] Vassalle M, Lee CO. The relationship among intracellular sodium activity, calcium, and strophanthidin inotropy in canine cardiac purkinje fibers. J Gen Physiol 1984;83:287307.

Address for correspondence:

Philip Stewart

Schuster Building

The University of Manchester

Manchester

M13 9PL

United Kingdom

philip.stewart@postgrad.manchester.ac.uk 